\title{
Data Driven Tools and Methods for Microtexture Classification and Dwell Fatigue Life Prediction in Dual Phase Titanium Alloys
}

\author{
V. Venkatesh, R. Noraas, A. Pilchak ${ }^{\$}$, S. Tamirisa ${ }^{++}$, K. Calvert $^{\wedge}$, A. Salem ${ }^{\#}$, T. Broderick ${ }^{\circledR}$, M.G. Glavicic $^{*}$, I. Dempster ${ }^{\$}$, V. Saraf $^{+}$
}

Pratt \& Whitney, East Harford CT

\$Air Force Research Lab, Dayton, OH

${ }^{+}$ATI Forged Products, Cudahy, WI

*Rolls-Royce Corporation, Indianapolis IN

$@_{G E}$ Aviation, Evandale, $\mathrm{OH}$

${ }^{\#}$ Materials Resources LLC, Dayton, OH

${ }^{++}$Arconic, Niles, Oh

${ }^{M}$ Titanium Metals Corporation, Henderson, NV

$\$ \$$ Wyman Gordon, Houston, TX

\begin{abstract}
$\underline{\text { Abstract }}$
Microtexture has been linked to large reductions in cold dwell fatigue (CDF) life of specific dual phase titanium alloy aeroengine components. A recently completed Metals Affordability Initiative (MAI) funded program led by Pratt \& Whitney (P\&W) and includes ATI Forged Products, Boeing, GE Aviation, Rolls Royce (RR), Arconic, Titanium Metals Corporation (TIMET), PCC-Wyman Gordon (PCC-WG), Scientific Forming Technologies (SFTC), Materials Resources LLC (MRL) and The Ohio State University (OSU) has developed improved techniques for the characterization of microtexured regions (MTR) in titanium billet and forgings, and integrated computational materials engineering (ICME). These methods are aimed at developing and integrating process and property modeling tools for the prediction of microtexture and fatigue life in titanium components. These characterization and fatigue life prediction tools have near-term application off ramps that will enable use for process and product development and quality control. Key results for two widely used alloys, Ti-6242 and Ti64 , will be reviewed in this paper.
\end{abstract}

\section{$\underline{\text { Introduction }}$}

The $a+b$ class of titanium alloys are widely used in the fan and compressor stages of jet engines, due to their excellent strength to weight ratio and corrosion resistance. However, near a and $\mathrm{a}+\mathrm{b}$ alloys such as Ti-6242 and Ti-6-4 have exhibited sensitivity to cold dwell fatigue, associated with cracking of microstructural regions that are unfavorably oriented for slip, also known as hard orientated grains [1-3].

Bache proposed a modified Stroh Model where dislocations pile ups in grains favorably oriented for slip, i.e., soft grains, result in stress concentrations along grain boundaries with neighboring hard oriented grains, ultimately initiating a crack [4]. Venkatramini, Ghosh and Mills later validated this proposal using a multi-scale computational finite element approach that identified a local phenomenon of load shedding, due to soft grains shedding load on to hard grains, with a significant rise in stress gradients across the interface [5]. Such stress redistribution between microtextural regions with different crystal orientations has been proposed as the fundamental cause of the development of cracks during dwell fatigue in near a titanium alloys. 
At the 1995 World Titanium conference, Woodfield et. al., presented their work on the relationship between microstructure, microtexture and dwell fatigue in $\mathrm{a}+\mathrm{b}$ processed Ti-6242 [6]. This seminal study used the electron backscatter diffraction (EBSD) technique to quantify microstructure and microtexture parameters effecting dwell fatigue life. The current study was aimed at evaluating the dwell fatigue sensitivity in $\mathrm{a}+\mathrm{b}$ alloys such as Ti-6-4 and developing and validating an open method for classifying and quantifying microtextured regions (MTR's) that can be utilized across the supply chain for the safe design and manufacture of titanium components.

This article provides a review of the Air Force funded, Metals Affordability Initiative (MAI) PW-9 program on "ICME of Microtexture Evolution and its Effect on Cold Dwell/High/Low Cycle Fatigue Behavior of Dual Phase Titanium Alloys", which is aimed at developing and integrating process and property modeling tools and methods for the characterization and prediction of microtexture and fatigue life in dual phase titanium alloy components.

\section{Experimental Procedure}

In order to build robust fatigue models that capture a range of commonly observed microstructures and microtextures, the AIPT utilized production scale and lab-scale Ti-6-4 and Ti- 6242 billet manufactured to various diameters. These billets were subsequently isothermally and non-isothermally forged at $50^{\circ} \mathrm{F}$ below the b-transus, to various upset reductions, followed by an a $+\mathrm{b}$ solution treatment and age that results in a primary a fraction between $30-55 \%$.

DEFORM-2D finite element (FE) forge simulations of the upset operations were conducted, and subsequently used to select fatigue sample blank locations. A total of 152 fatigue blanks -from Ti-6-4 pancakes and Ti-6242 pancakes - were extracted, machined, and peened. The fatigue samples were peened using S70 steel shot at an intensity between 45 to 85 psi. Dwell low cycle fatigue (DLCF) and continuous low cycle fatigue (LCF) test coupon pairs were extracted from every major strain level contour in the pancakes, as shown in Figure 1. This ensured a good distribution of MTR sizes and intensity levels in the fatigue samples. All the fatigue tests were run at an R=0 and maximum steady stress levels ranging between 120 to $132 \mathrm{KSI}$, with the addition of a 2 minute tensile dwell time added to the DLCF tests. In addition, a total of 74 room temperature tensile tests were also conducted according to ASTM E8 from blanks extracted from these Ti-6-4 and Ti-6242 pancakes.

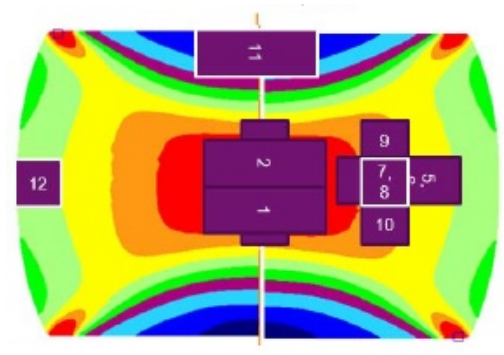

Figure 1. DEFORM-2D FE simulation of a forged pancake, color contours representing effective strain levels, which were used to select fatigue coupon locations depicted as rectangular shaded boxes.

In order to adequately capture the morphological and texture characteristics of microtextured regions within the samples it was necessary to capture large scans covering several square millimeters. Electron backscatter diffraction (EBSD) was conducted on DLCF samples, sectioned along the longitudinal axis, to produce inverse pole figure maps and pole figures as well as texture data for subsequent MTR identification and quantification. A step size of $5 \mu \mathrm{m}$ was used to balance resolution of microtextured regions with scan duration. A few of these scans are summarized in Figure 2. For reference, the tensile stress axis runs horizontally through the images referred to as the [010] IPF direction. Significant qualitative differences were observed in the overall level of microtexture present. 

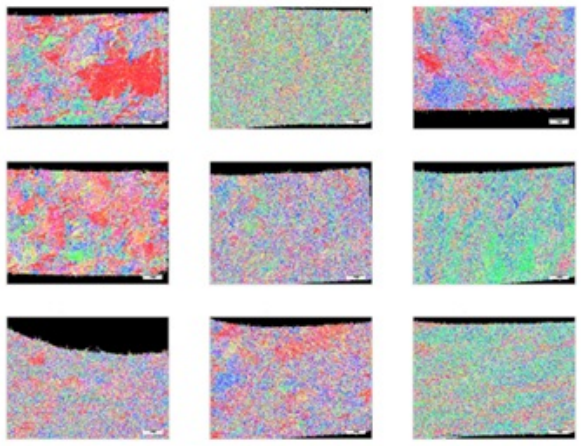
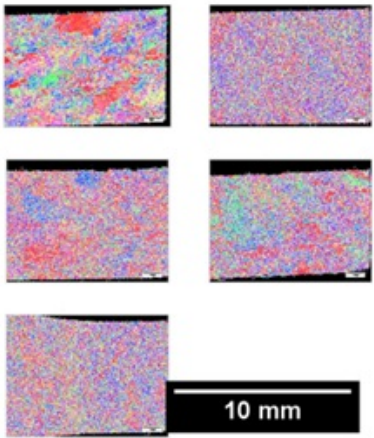

Figure 2. Electron Back Scatter Diffraction (EBSD) Images for a few Ti-6-4 LCF samples, sectioned along the longitudinal axis ([010] IPF direction).

A major project goal was to develop tools and methods (T\&M's) for the industrial supply chain to use for identification and quantification of MTR's. Two T\&M's were developed that utilized the large-area EBSD data detailed previously. The first T\&M applied MRL's commercial software, TiZone ${ }^{\mathrm{TM}}$, for the identification of MTRs through successive clustering of data points by orientation and spatial location, followed by the segmentation of identified MTRs into orientation "classes" based on C-axis orientation and finally generating metrics based on n-point statistics of each MTR class, including size, aspect ratio, and area fraction. MRL's methods were described in detail at the 13th Ti-World conference in San Diego [7].The second T\&M utilized the open source software, Dream3D, with program developed pipelines to import and clean EBSD data, segment features based on pixel misorientation, and store the data in an efficient HDF5 format. The Dream3D software by itself outputs metrics like feature size, average misorientation, Euler angles, etc. MATLAB developed postprocessing scripts were subsequently used to compute additional metrics, like feature stress axis misalignment, density, intensity, degree of spatial clustering, and hard/soft/initiator class labels, among others (Figure 3). The standard MTR parameters and values used for the PW9 program are shown in Table 1, which highlights the minimum size of an MTR, as well as the stress-axis misalignment values used for classification of hard, soft, and initiator MTRs. In addition, a single factor called MTR intensity, described in Equation (1), that links three other parameters was used to compare the severity of microtexture levels between various material datasets manufactured in this program.

$$
\mathrm{I}_{\mathrm{MTR}}=\frac{\rho \cdot A}{\Delta C}
$$

where $\mathrm{I}_{\mathrm{MTR}}$ is the MTR intensity, $\mathrm{r}$ is the density of similarly aligned alpha grains in an MTR, A is the MTR size and DC is the c-axis misalignment within an MTR. 


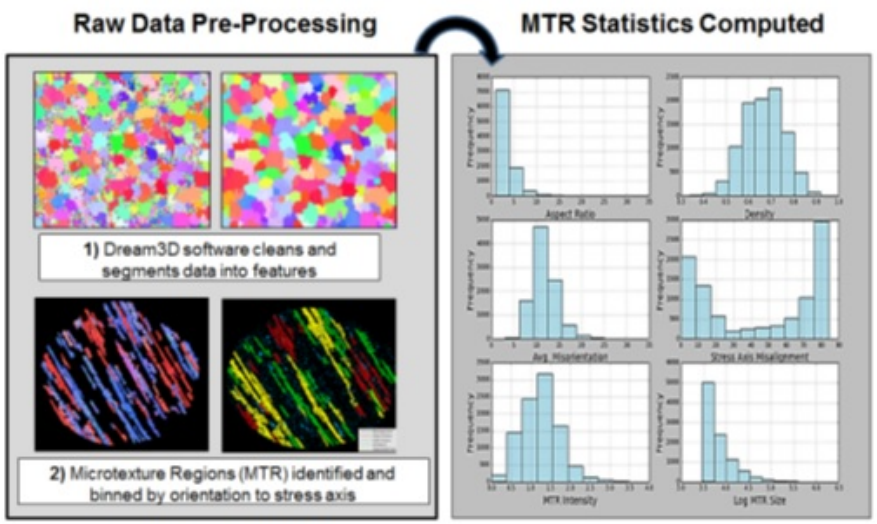

Figure 3. Schematic Illustration of Microtexture Quantification Framework Using Dream3D and Matlab

Table 1. Dream3D Based MTR Parameters and Standard Values Used in PW9 Program

\begin{tabular}{|c|c|}
\hline DREAM 3D Parameter & Value: \\
\hline \hline Min EBSD Confidence Index & $\mathbf{0 . 2 0}$ \\
\hline Segment Features (C-axis Misalignment) & $20 \mathrm{deg}$ \\
\hline Minimum MTR Size & $10,000 \mathrm{~mm}^{2}$ \\
\hline Hard MTR Classification & $\{10-17\}<25$ deg of stress axis \\
\hline Soft MTR Classification & {$[0001]$ within $70-90$ deg of stress axis } \\
\hline Initiator MTR Classification & {$[0001]$ within $40-50$ deg of stress axis } \\
\hline
\end{tabular}

\section{$\underline{\text { Results and Discussion }}$}

LCF and DLCF results for Ti-6242 and Ti-6-4, shown in Figure 4, indicate a tendency for increased cyclic life in material containing lower microtexture levels. Analysis of the datasets suggests that a combination of good billet processing with higher forging strains result in higher fatigue lives. However, no strong effect of yield strength on fatigue life of Ti-6-4 and Ti-6242 was observed (Figure 5). These results suggest a complex inter-relationships between MTR metrics, test conditions, microstructure characteristics and dwell fatigue life of both alloys that were subsequently identified using machine learning tools. In addition, it was observed that dwell fatigue life of Ti-6-4 tested at equivalent percent yield strength levels were much shorter than for Ti-6242. This behavior is consistent with lower creep capability of Ti-6-4 compared with Ti-6242. 


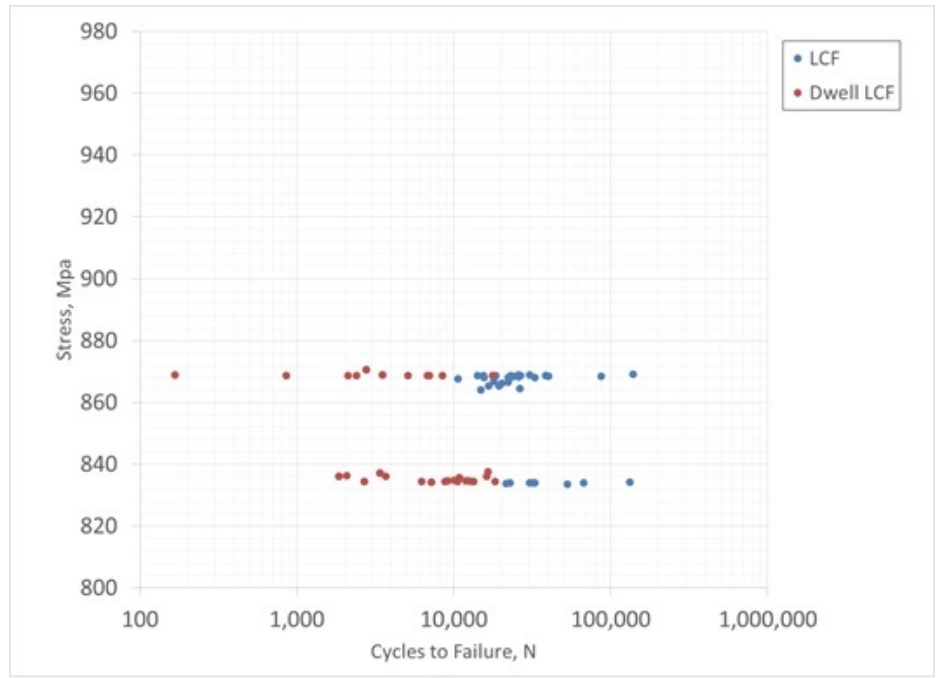

(a)

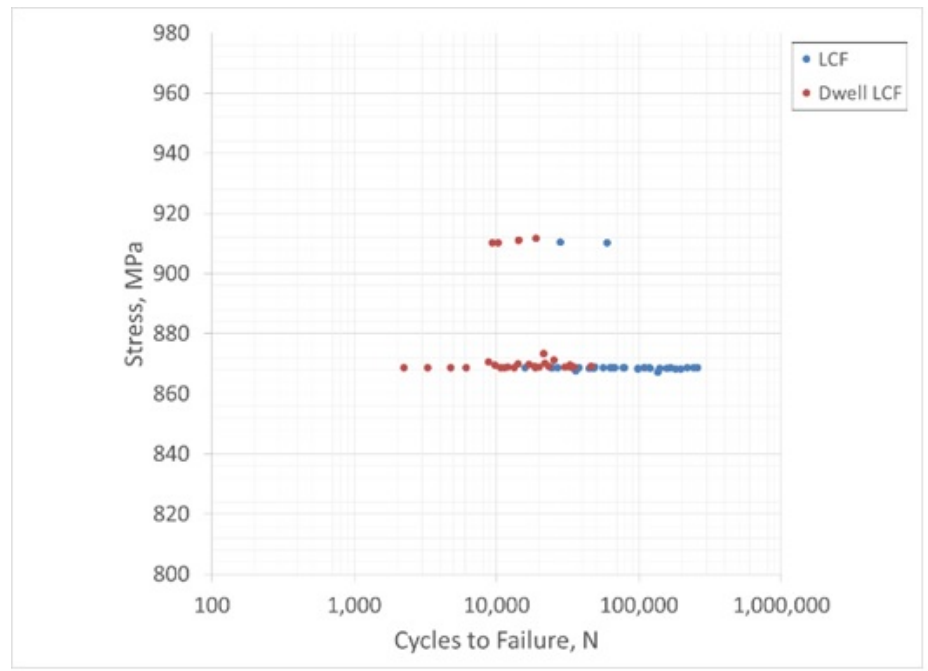

(b)

Figure 4. DLCF and LCF results for (a) Ti-6-4 and (b) Ti-6242. Continuous LCF points are in blue and dwell LCF points are shown in red. 


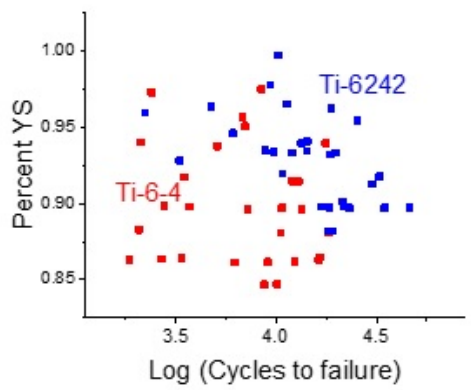

Figure 5. Dwell fatigue lives in log scale of Ti-6-4 and Ti-6242 Samples Tested at stress levels as a function of percent tensile yield strength (YS).

Box plots of microtexture metrics were compiled for all MTRs greater than $10,000 \mathrm{um}^{2}$, and were broken down by material process path (A to D), Figure 6. It is interesting to note quantifiable differences for MTR sizes and intensities between material process paths, which in-turn could enable quantified manufacturing process parameter requirements.
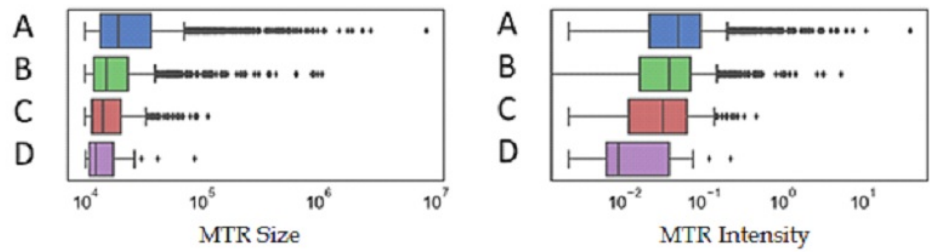

(a)
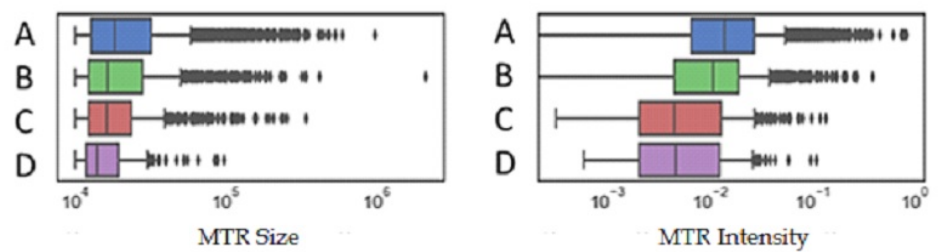

(b)

Figure 6. Comparison of MTR Size and Intensity for (a) Ti-6-4 and (b) Ti-6242 LCF Samples between billet and forging path combinations (high/low strain combinations), identified in the vertical axis as A, B, C and D.

Eureqa ${ }^{(\mathrm{R})}$, a commercially available software that automatically builds models, was used to calibate and validate a model that explicitly captured the influence of MTR metrics on fatigue life. A total of 59 DLCF tests were used to develop life models in Eureqa - 80 percent of the data was used for model training and 20 percent for model validation. Over 70 statistically based microstructure and microtexture parameters along with the maximum stress as a function of yield strength were used to identify the critical parameters that correlate with dwell fatigue life for these 59 datasets. Eureqa splits datasets into a training dataset and a validation dataset in order to perform cross validation and prevent overfitting. Training data is used to generate and optimize solutions, and the validation set is used to test how well those models generalize to new data.

A total of five Eureqa models that had similar model errors were chosen for predicting sample dwell life. The mathematical relationships in these models were automatically generated by Eureqa, each having slightly different formulations and generalization capability over the testing range. Eureqa successfully identified 3 MTR metrics, along with the percent yield strength of the dwell test, as critical model inputs for DLCF life prediction (Figure 7). These results suggest that higher test stress levels and larger soft MTR sizes 
negatively impact DLCF life. In contrast, a larger fraction of soft MTRs and miscellaneous grains positively influence DLCF life. The significance of large soft MTRs is supported by many studies on dwell fatigue behavior in titanium alloys, which suggest that high stresses are developed at the interface between large soft MTRs that accommodate slip and hard MTRs that do not deform, leading to crack initiation and life shortfalls. On the other hand, having a large fraction of soft and randomly oriented grains will help to homogenize the strain distribution and improve life. All five Eureqa models were chosen for comparing sample dwell life prediction with measured failure life cycles. Figure 8 shows that all the predictions, except one, were within a $2 \mathrm{X}$ life bounds. This Ti-6-4 outlier had an extremely low life but contained average MTR metrics.

\begin{tabular}{|l|r|l|}
\hline \multicolumn{1}{|c|}{ Variable Name } & $\begin{array}{c}\text { \% Impact on } \\
\text { Model }\end{array}$ & $\begin{array}{c}\text { Model Sensitivity } \\
\text { to Variable }\end{array}$ \\
\hline Misc Alpha Grain Fraction & $51.00 \%$ & \\
\hline Mean Soft MTR Size & $-100.00 \%$ & \\
\hline Soft MTR Fraction & $100.00 \%$ & \\
\hline Fraction of Yield Strength & $-100.00 \%$ & \\
\hline
\end{tabular}

Figure 7. List of Eureqa Model Input Parameters Along With Their Sensitivity to DLCF

Once the critical MTR model input parameters were identified and DLCF life model were generated, Eureqa was used to develop models linking forge parameters to MTR evolution from billet to pancakes. This was accomplished by using starting billet MTR metrics together with forging temperature and location based effective strains that correspond to DLCF samples in the pancake. The strain levels were previously determined using DEFORM simulations of the pancake process. Results of predictions showed that about 10 of 59 predicted datasets lay outside the $2 \mathrm{X}$ life bounds.

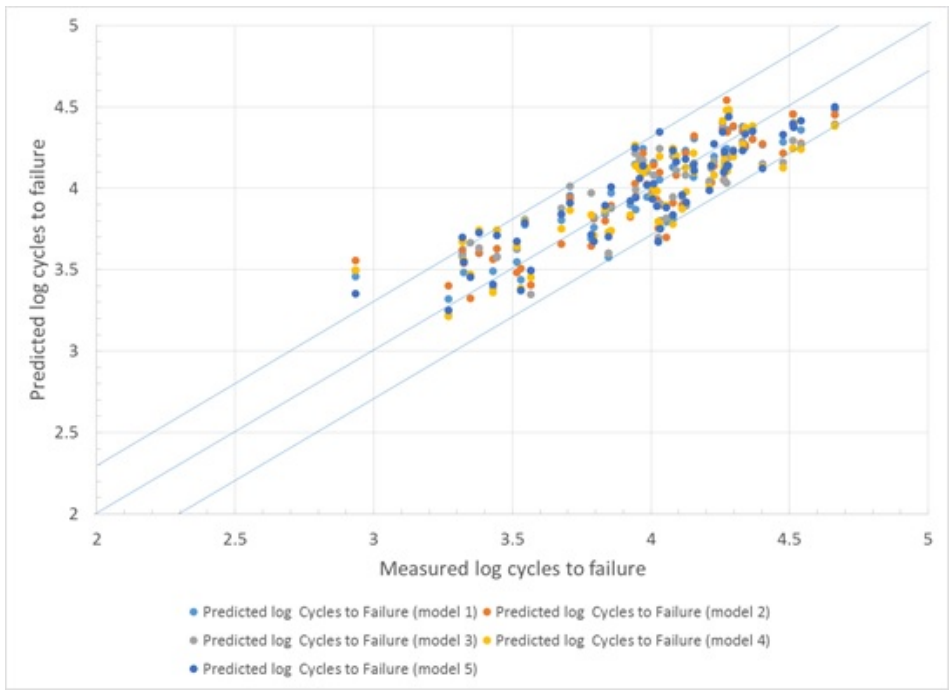

Figure 8. Comparison of DLCF Predictions Using Eureqa based Dwell Life Models for Ti-6242 and Ti-6-4. With the exception of one sample data point, all the predictions lie within a $2 \mathrm{X}$ life bounds.

\section{Summary}

The PW-9 program successfully developed critical tools and methods for implementation in the supply chain. These tools will support the design of manufacturing processes and final components, as described below:

- EBSD based tools and methods to identify, classify and quantify MTRs

- Large datasets comprising of area scans spanning $8 \mathrm{~mm} \times 8 \mathrm{~mm}$ were used to capture MTRs. An open source software, Dream3D, was utilized to import and clean EBSD data, segment features based on pixel misorientation, and store the data in 
an efficient format. MATLAB post-processing scripts were subsequently used to compute additional MTR classification and quantification metrics.

- Accurate Dwell fatigue life models for Ti-6-4 and Ti-6-2-4-2

- Fatigue models for Ti-6-4 and Ti-6-2-4-2 were successfully validated using fatigue test parameters, microstructure and microtexture metrics. Mean life predictions were within a factor of 2, and within the 95 percent confidence of the experimentally determined mean fatigue life.

- Analytic models that explicitly capture the influence of MTR metrics on fatigue life were also developed utilizing Eureqa. This approach captured three critical MTR characteristics that significantly impact DLCF.

\section{Acknowledgements}

The authors would like to acknowledge the support of the U.S. Air Force through the Metal Affordability Initiative (contract FA8650-13-25203), and team members; S.L. Semiatin, I. Cernatescu, R. Shankar.

\section{$\underline{\text { References }}$}

[1] P.D. Littlewood, A. J. Wilkinson, Int. Journal Fatigue, (2012) 111-119.

[2] D. Lunt, "The Effect of Macrozones in Ti-6Al-4V on Strain Localization Behavior, PhD Thesis, University of Manchester, 2014.

[3] G. Lutjering, J. C. Williams, Titanium, Springer Berlin Heidelberg New York, $2^{\text {nd }}$ edition, 2007.

[4] M. R. Bache, Int. J. Fatigue, 25, 2008 1079-1087

[5] G. Venkatramini, S. Ghosh, M. Mills, Acta Materialia, 55, 2007, 3971-3936

[6] A.P. Woodfield, M. D. Gorman, R.R. Corderman, J.A. Sutliff, B. Yamron, Titanium 95, Science and Technology, The University Press, Cambridge, UK, 1996.

[7] V. Venkatesh, S. Tamirisa, J. Sartkulvanich, K. Calvert, I. Dempster, V. Saraf, A.A. Salem, S. Rokhlin, T. Broderick, M.G. Glavicic, T. Morton, R. Shankar, A. Pilchak, "ICME of Microtexture Evolution in Dual Phase Titanium Alloys", Proceedings of 13th World Titanium Conference, V. Venkatesh, et. al. eds, 2015. 